\title{
Nash Bargaining in Ordinal Environments
}

\author{
By Özgür Kıbrı*
}

December 17, 2004

\begin{abstract}
We analyze the implications of Nash's (1950) axioms in ordinal bargaining environments; there, the scale invariance axiom needs to be strenghtened to take into account all order-preserving transformations of the agents' utilities. This axiom, called ordinal invariance, is a very demanding one. For two-agents, it is violated by every strongly individually rational bargaining rule. In general, no ordinally invariant bargaining rule satisfies the other three axioms of Nash. Parallel to Roth (1977), we introduce a weaker independence of irrelevant alternatives axiom that we argue is better suited for ordinally invariant bargaining rules. We show that the three-agent Shapley-Shubik bargaining rule uniquely satisfies ordinal invariance, Pareto optimality, symmetry, and this weaker independence of irrelevant alternatives axiom. We also analyze the implications of other independence axioms.
\end{abstract}

*Faculty of Arts and Social Sciences, Sabancı University, Orhanlı, 81474 Tuzla, Istanbul, Turkey. Fax: +90-216-483-9250. E-mail: ozgur@sabanciuniv.edu 


\section{Introduction}

In his seminal work, Nash (1950) postulates four axioms that he argues solutions to bargaining problems do satisfy: scale invariance, Pareto optimality, symmetry, and independence of irrelevant alternatives. He proves that only one bargaining rule, now commonly known as the Nash bargaining rule, satisfies these axioms.

In this paper, we analyze the implications of Nash axioms in ordinal environments, that is, in bargaining situations where the agents' preferences are only restricted to be complete, transitive, and continuous. For ordinal environments, the scale invariance axiom of Nash is not sufficient to ensure the consistency of the bargaining solution (or equivalently, the invariance of the physical bargaining outcome) with respect to changes in the utility representation of the agents' underlying preferences. It needs to be replaced with a stronger axiom called ordinal invariance. Unfortunately, the Nash bargaining rule violates this axiom.

The previous literature on ordinal bargaining is quite small. Shapley (1969) shows that for two agents, no strongly individually rational bargaining rule satisfies ordinal invariance. However, it later turns out that this negative result is limited to two agents; an ordinally invariant and strongly individually rational bargaining rule for three agents appears in Shubik (1982). We will refer to it as the Shapley-Shubik rule. ${ }^{1}$

For Pareto surfaces with more than two agents, Sprumont (2000) proves the existence of an ordinal basis, that is, a subclass of surfaces which, through order-preserving transformations, generates all Pareto surfaces, and which is minimal. Furthermore, he constructs a "sufficiently symmetric" ordinal basis for three-agent surfaces. Kıbris (2004) uses Sprumont's construction to describe a class of three-agent "ordinally normalized problems" which, through order-preserving transformations of the agents' utilities, generates all bargaining problems. $^{2}$ On this class, the Shapley-Shubik rule coincides with the Egalitarian (Kalai,

\footnotetext{
${ }^{1}$ There is no reference on the origin of this rule in Shubik (1982). However, Thomson [16] attributes it to Shapley. Furthermore, Roth (1979), in pages 72-73, mentions a three-agent ordinal bargaining rule proposed by Shapley and Shubik (1974, Rand Corporation, R-904/4) which, considering the scarcity of ordinal rules in the literature, is most probably the same bargaining rule.

${ }^{2}$ The class of ordinally normalized problems can be interpreted as the ordinal counterpart of the class of $0-1$ normalized problems (that is, agents' disagreement payoffs are normalized to 0 and aspiration payoffs
} 
1977) and the Kalai-Smorodinsky (1975) rules since it always chooses the maximizer of a Leontief type order. Kıbris also shows that the Shapley-Shubik rule is the only symmetric member of a class of (ordinal) monotone path rules and that these are the only ordinally invariant rules that are Pareto optimal and "monotonic".

For problems with more than three agents, our knowledge is quite restricted. It follows from Sprumont (2000) that for such problems ordinally invariant, Pareto optimal, and strictly individually rational bargaining rules exist. Furthermore, Safra and Samet (2001a, 2001b) propose two generalizations of the Shapley-Shubik formula to this case. However, since these formulas (and in general, formulas defining non-dictatorial ordinal rules) are quite complicated, ${ }^{3}$ construction of a class of ordinally normalized problems (over which the rule under question has a simpler definition) significantly helps to facilitate an axiomatic analysis. ${ }^{4}$ Such a construction does not yet exist for more than three agents. ${ }^{5}$ It is for this reason that the analysis of this paper is restricted to the three-agent case.

Ordinally invariant rules do not satisfy all of the other three axioms of Nash (1950). Among them we consider Pareto optimality and symmetry to be more basic. We therefore look for ordinally invariant, Pareto optimal, and symmetric rules that satisfy a weaker form of independence of irrelevant alternatives (IIA): the original axiom of Nash (1950) requires that the solution to a bargaining problem should not change as some of the alternatives (other than the original solution) cease to be feasible; note that the problem's disagreement point remains unchanged. IIA has been frequently criticized on the basis that it requires the bargaining rule to be too insensitive to changes in the set of feasible utility profiles (e.g. see Luce and Raiffa, 1957). Based on this criticism, Roth (1977) proposes a

to 1) for cardinal bargaining rules. Both classes have the property that any physical bargaining problem has a utility image in this subclass (and therefore, any problem outside the class is equivalent to a member of this class).

${ }^{3}$ The Shapley-Shubik and the Safra-Samet solutions to arbitrary bargaining problems are defined as the limit of a sequence constructed on the problem's Pareto surface.

${ }^{4}$ For example, Kibris (2001) utilizes the fact that on the class of ordinally normalized problems, the Shapley-Shubik rule coincides with the Egalitarian rule.

${ }^{5}$ Note that the issue is not the existence of a normalized class but that of constructing one that is desirable in the aforementioned sense. In fact, Sprumont presents a highly asymmetric construction for more than three agents and notes that "it may be of little use to define attractive solutions". 
weakening of the axiom appropriate for cardinal bargaining environments; this axiom, called independence of irrelevant alternatives other than the aspiration points (IIAaspiration), requires that the problem's aspiration point (additional to the disagreement point) remain unchanged as the feasible set contracts. Since an affine transformation with two fixed points can only be the identity mapping, IIA-aspiration effectively rules out the possibility of comparing two alternative (cardinal) utility representations of the same physical problem. Also note that IIA-aspiration is the restriction of IIA to a "cardinal basis" of bargaining problems, namely the class of $0-1$ normalized problems.

For Pareto optimal rules, the scale invariance and IIA properties do not make contradictory statements about how solutions to problem pairs should be related. ${ }^{6}$ This however is not the case when scale invariance is replaced with ordinal invariance (e.g. see Example 4). Due to the nonlinear transformations allowed, it becomes essential to separate the jurisdictions of ordinal invariance from a compatible IIA property. Thus we repeat the exercise of Roth (1977) for ordinal rules and observe that restricting the comparison to problems which have a common set of Pareto optimal points, called the "extended brace", rules out the possibility of comparing two alternative (ordinal) utility representations of the same physical problem. Motivated by this observation, we propose independence of irrelevant alternatives other than the extended brace (IIA-extended brace) which only considers contractions of the feasible set in which the extended brace of the original problem (additional to the original solution and the disagreement point) remain feasible. As in IIAaspiration, this axiom is nothing but a restriction of Nash's IIA property to an "ordinal basis" of bargaining problems that will be introduced in Section 2.

In Section 2, we demonstrate that the extended brace of a problem is constructed via a set of Pareto optimal points which we call the "brace". The aspiration points of a problem summarize what each agent can achieve if he dictates the decision taken in the bargaining process. The brace points have a similar interpretation; they generalize the idea of

\footnotetext{
${ }^{6}$ Without Pareto optimality, the two requirements can contradict. Consider for example $d=(0,0)$, $S=\operatorname{conv}\{(0,0),(1,0),(0,1)\}$ and $S^{\prime}=\operatorname{conv}\left\{(0,0),\left(\frac{1}{2}, 0\right),\left(0, \frac{1}{2}\right)\right\}$. The problems $(S, d)$ and $\left(S^{\prime}, d\right)$ are related to each other through both an affine transformation of the agents utilities and by a contraction of $S$ to $S^{\prime}$. For example if $F(S, d)=\left(\frac{1}{4}, \frac{1}{4}\right)$, scale invariance requires $F\left(S^{\prime}, d\right)=\left(\frac{1}{8}, \frac{1}{8}\right)$ and IIA requires $F\left(S^{\prime}, d\right)=\left(\frac{1}{4}, \frac{1}{4}\right)$.
} 
dictatorship from individuals to coalitions (for further discussion on the "extended brace" and the "brace", see Kıbris (2004)). We thus also propose a stronger version of the above property called independence of irrelevant alternatives other than the brace (IIAbrace). It requires the brace of the original problem (additional to the original solution and the disagreement point) remain feasible in a contraction of the feasible set. As will be discussed below, the relation between these two properties resemble that between IIA and IIA-aspiration.

We first show that a class of ordinal rules, including the Shapley-Shubik rule satisfies $I I A$-extended brace and all of these rules coincide on a large class of bargaining problems. We next show that the Shapley-Shubik bargaining rule uniquely satisfies ordinal invariance, Pareto optimality, symmetry, and IIA-brace. These results are of interest for three reasons. First, they suggests that similar axioms lead to two different practices in cardinal and ordinal approaches to bargaining: while the product of the agents' utility gains is maximized in the former, the "utility" of the worst-off agent is maximized in the latter. Second, when analyzed in relation to the findings of Kibris (2004), these results suggest that the Shapley-Shubik rule is the ordinal counterpart of both the Nash and the Kalai-Smorodinsky bargaining rules which, in the cardinal approach seem to be based on different principles. ${ }^{7}$ Finally, this observation might provide some intuition on explaining why equal division is the most prominent outcome in bargaining experiments: this is what the Shapley-Shubik rule proposes for any three-agent bargaining problem on the allocation of a single divisible good (such as money). However, we are not aware of any three-agent experiment at this point and the conclusions of the Shapley-Shubik rule only apply to this case.

Finally we show that IIA-brace is the strongest axiom of its kind which is satisfied by ordinally invariant, Pareto optimal, and symmetric bargaining rules. Furthermore, such rules all violate the IIA-aspiration axiom of Roth (1977).

\footnotetext{
${ }^{7}$ Interestingly enough, the ordinal rule of O'Neill et al (2001) has a similar property.
} 


\section{Model}

Let $N=\{1,2,3\}$ be the set of agents. Vector inequalities are written as $\leqq, \leq$, and $<$. For each $i \in N, e(i)$ stands for the vector in $\mathbb{R}_{+}^{N}$ whose $i$ th coordinate is 1 and all other coordinates are 0 . Let $\Pi$ be the set of all permutations $\pi$ of $N$. For each $\pi \in \Pi, x \in \mathbb{R}^{N}$, and $S \subset \mathbb{R}^{N}$, let $\pi(x)=\left(x_{\pi(i)}\right)_{i \in N}$ and $\pi(S)=\{\pi(y): y \in S\}$. For each $X \subset \mathbb{R}^{N}$ and $x \in \mathbb{R}^{N}$, $\operatorname{conv}\{X\}$ is the convex hull of $X$ and $\operatorname{comp}\{X \mid x\}=\left\{y \in \mathbb{R}^{N}: y \geqq x\right.$ and $y \leqq z$ for some $z \in X\}$ is the comprehensive hull of $X$ down to $x$.

A pair $(S, d) \in 2^{\mathbb{R}^{N}} \times \mathbb{R}^{N}$ is a bargaining problem if $(i) S$ is compact, $(i i) d \in S$, and (iii) there is $x \in S$ with $x>d$. A bargaining problem $(S, d)$ is strictly d-comprehensive if for each $x \in S$ and $y \in \mathbb{R}^{N}$ such that $d \leq y \leq x, y \in S$ and there is $z \in S$ such that $z>y$. This axiom has two implications: first, that utility is disposable down to the disagreement point; second, that any individually rational and weakly Pareto optimal point is also Pareto optimal. Let $\mathcal{B}$ denote the set of all strictly $d$-comprehensive bargaining problems.

For each $(S, d) \in \mathcal{B}$ and $i \in N$, agent $i^{\prime} s$ aspiration payoff is $a_{i}(S, d)=\max \left\{s_{i}:\left(s_{i}, d_{-i}\right) \in\right.$ $S\}$ and his aspiration point is $\operatorname{asp}_{i}(S, d)=\left(a_{i}, d_{-i}\right)$. Let $P(S, d)$ denote the set of Pareto optimal profiles in $\mathbf{S}: x \in P(S, d)$ if and only if $x \in S$ and for each $y \geq x, y \notin S$. Let $I(S, d)$ denote the set of individually rational profiles in $\mathbf{S}: x \in I(S, d)$ if and only if $x \in S$ and $x \geqq d$. Let $I P(S, d)$ denote the set of Pareto optimal and individually rational profiles in $\mathbf{S}$. Let $\mathcal{B}_{I}=\{(S, d) \in \mathcal{B} \mid S=I(S, d)\}$.

For each $i \in N$, let $f_{i}$ be an increasing continuous function on $\mathbb{R}$ and define $f=\left(f_{i}\right)_{i \in N}$ : $\mathbb{R}^{N} \rightarrow \mathbb{R}^{N}$ by $f(x)=\left(f_{i}\left(x_{i}\right)\right)_{i \in N}$ for each $x \in \mathbb{R}^{N}$. Let $\mathcal{F}$ denote the set of such functions. Two problems $(S, d),\left(S^{\prime}, d^{\prime}\right) \in \mathcal{B}$ are ordinally equivalent if there is $f \in \mathcal{F}$ such that $f(S)=S^{\prime}$ and $f(d)=d^{\prime}$. Otherwise, they are called ordinally distinct. A subclass $\mathcal{B}^{\prime} \subseteq \mathcal{B}$ ordinally spans $\mathcal{B}$ if for any problem $(S, d) \in \mathcal{B}$, there is a problem $\left(S^{\prime}, d^{\prime}\right) \in \mathcal{B}^{\prime}$ which is ordinally equivalent to it. The subclass $\mathcal{B}^{\prime}$ is an ordinal basis of $\mathcal{B}$ if it ordinally spans $\mathcal{B}$ and all problems in $\mathcal{B}^{\prime}$ are ordinally distinct. 


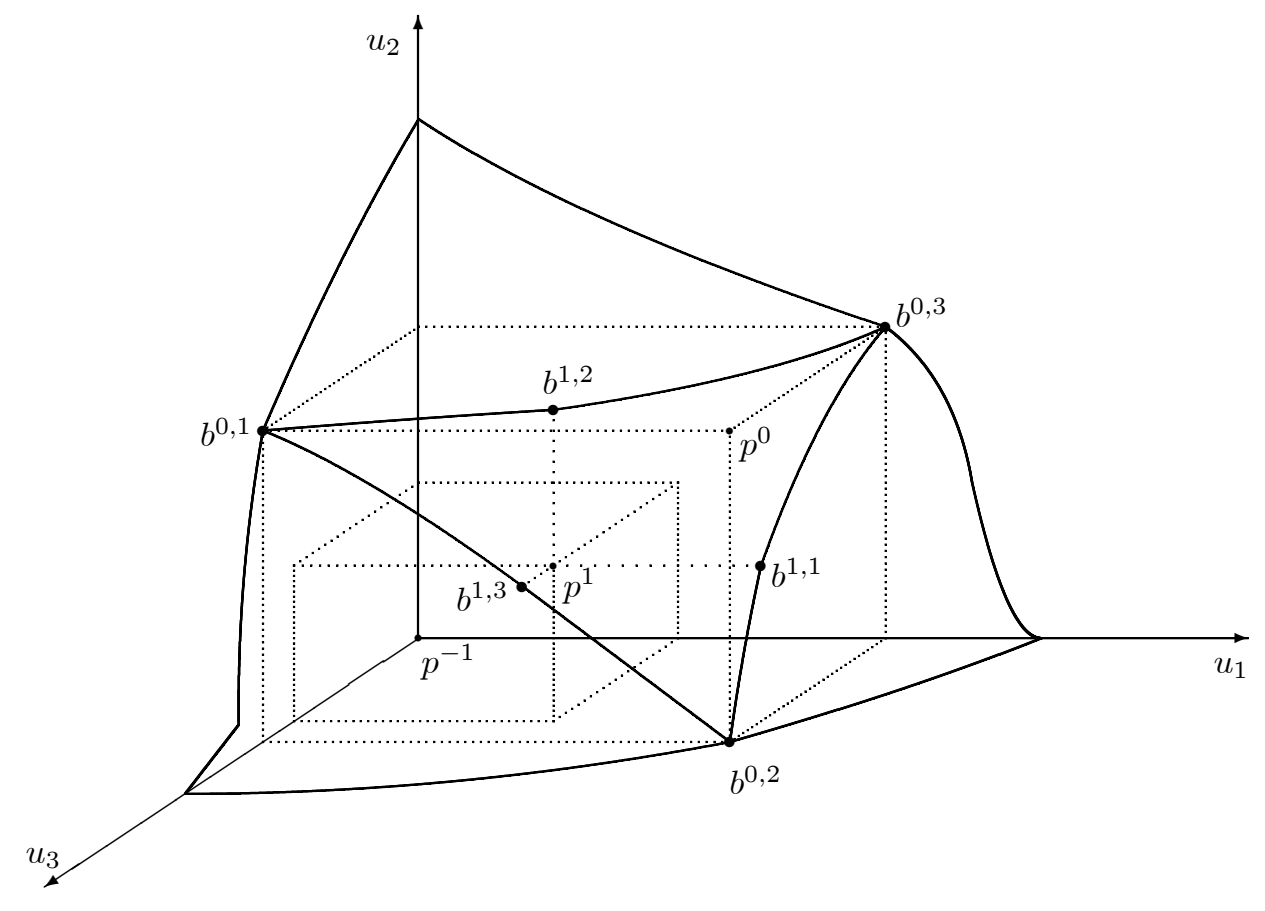

Figure 1: Constructing the brace of an arbitrary problem.

\subsection{Brace and ordinally normalized problems}

Let $(S, d) \in \mathcal{B}$. Define $p^{-1}(S, d)=d$ and for each $n \in \mathbb{N}$ define $p^{n}(S, d) \in \mathbb{R}^{N}$ to be such that

$$
\begin{aligned}
& b^{n, 1}(S, d):=\left(p_{1}^{n-1}(S, d), p_{2}^{n}(S, d), p_{3}^{n}(S, d)\right) \in P(S, d), \\
& b^{n, 2}(S, d):=\left(p_{1}^{n}(S, d), p_{2}^{n-1}(S, d), p_{3}^{n}(S, d)\right) \in P(S, d), \text { and } \\
& b^{n, 3}(S, d) \quad: \quad=\left(p_{1}^{n}(S, d), p_{2}^{n}(S, d), p_{3}^{n-1}(S, d)\right) \in P(S, d) .
\end{aligned}
$$

The sequence $\left\{p^{n}(S, d)\right\}_{n \in \mathbb{N}}$ is uniquely defined and it is convergent. Also note that for each $i \in N, \lim _{n \rightarrow \infty} p^{n}(S, d)=\lim _{n \rightarrow \infty} b^{n, i}(S, d)$. The brace of $(S, d)$ is a subset of $I P(S, d)$ and it is defined as

$$
\left.\operatorname{br}(S, d)=\bigcup_{n \in \mathbb{N}} \bigcup_{i \in N}\left\{b^{n, i}(S, d)\right\} . \quad \text { (see Figure } 1\right)
$$

We use the brace to define a subclass of $\mathcal{B}$. Let $(S, d) \in \mathcal{B}$ and define $b^{-1,1}(S, d):=$ $\left(\operatorname{asp}_{1}(S, d), d_{2}, d_{3}\right), b^{-1,2}(S, d):=\left(d_{1}, a_{s p}(S, d), d_{3}\right)$, and $b^{-1,3}(S, d):=\left(d_{1}, d_{2}, a s p_{3}(S, d)\right)$. Note that $\left\{b^{-1, i}(S, d)\right\}_{i \in N}$ are not brace points but aspiration points. For each $n \in \mathbb{N}$ and $i \in N$, let $A^{n, i}(S, d)$ be the Pareto optimal curve that connects $b^{n-1, i}(S, d)$ and $b^{n, i+1}(S, d)$ 


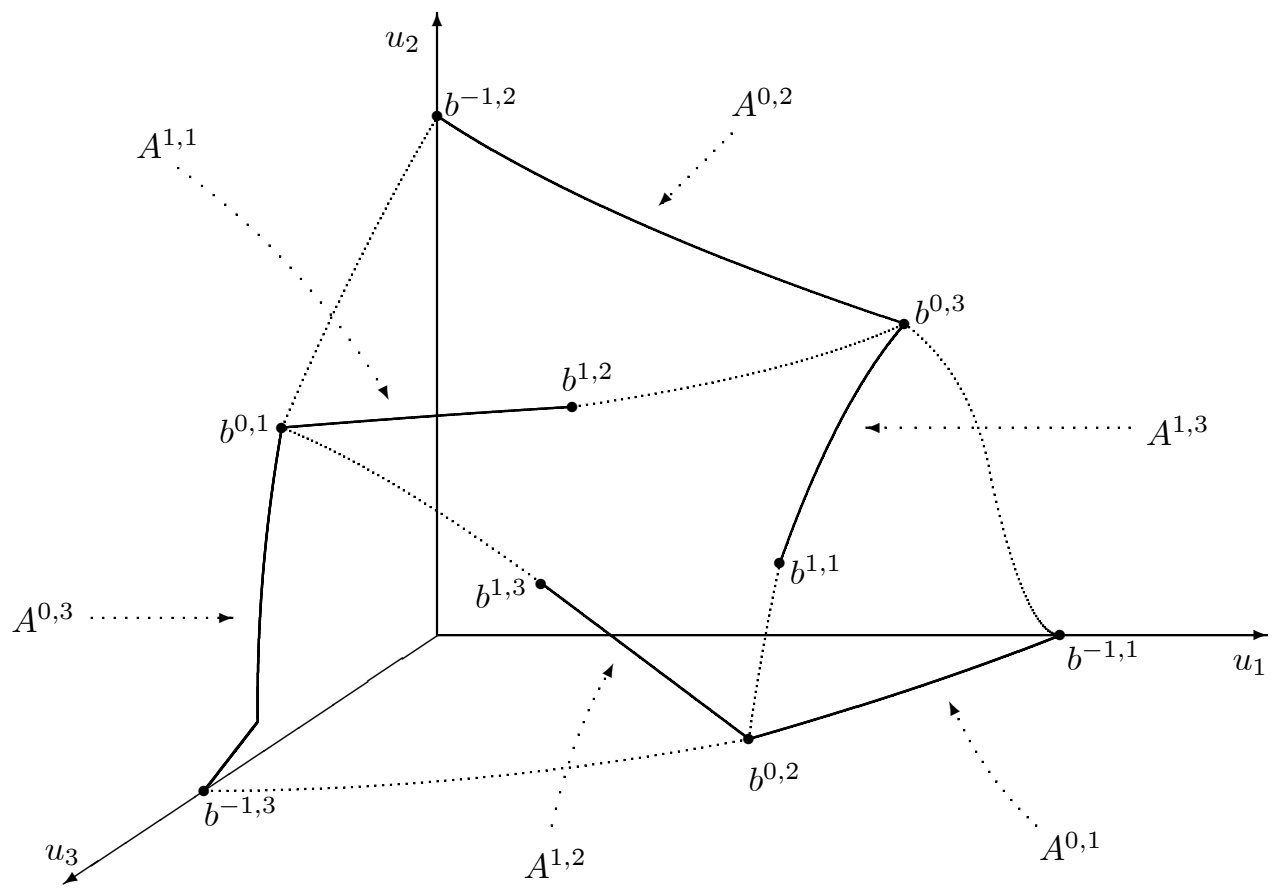

Figure 2: Constructing the extended brace of an arbitrary problem.

(with the convention that for $i=3, i+1=1$ ) as follows:

$$
A^{n, i}(S, d)=\left\{\begin{array}{c}
x \in P(S, d): \text { for each } j \in N, \\
\min \left\{b_{j}^{n-1, i}(S, d), b_{j}^{n, i+1}(S, d)\right\} \leqq x_{j} \leqq \max \left\{b_{j}^{n-1, i}(S, d), b_{j}^{n, i+1}(S, d)\right\}
\end{array}\right\}
$$

The extended brace of $(S, d)$ is (see Figure 2)

$$
A(S, d)=\bigcup_{n \in \mathbb{N}} \bigcup_{i \in N} A^{n, i}(S, d) .
$$

For bargaining problems whose set of individually rational and Pareto optimal points coincide with the unit simplex, the brace and the extended brace are trivially defined. Let $d^{*}=0$ and $S^{*}=\operatorname{comp}\left\{\operatorname{conv}\{e(1), e(2), e(3)\} \mid d^{*}\right\}$. Then, $p^{0}\left(S^{*}, d^{*}\right)=\left(\frac{1}{2}, \frac{1}{2}, \frac{1}{2}\right), p^{1}\left(S^{*}, d^{*}\right)=$ $\left(\frac{1}{4}, \frac{1}{4}, \frac{1}{4}\right)$, and for $n \geq 2, p^{n}\left(S^{*}, d^{*}\right)=\frac{1}{2}\left(p^{n-1}(S, d)+p^{n-2}(S, d)\right)$. This implies that for each $i \in$ $N, b^{-1, i}\left(S^{*}, d^{*}\right)=e(i)$ and for each $n \in \mathbb{N}, b^{n, i}\left(S^{*}, d^{*}\right)=\frac{1}{2}\left(b^{n-1, i-1}\left(S^{*}, d^{*}\right)+b^{n-1, i+1}\left(S^{*}, d^{*}\right)\right)$. That is, each brace point of this problem is obtained as the midpoint average of two other brace points and the averaging starts with the unit vectors. The normalized extended brace, $A^{*}$, is defined as the extended brace of this problem: $A^{*}=A\left(S^{*}, d^{*}\right)$. A bargaining problem $(S, d) \in \mathcal{B}$ is ordinally normalized (see Figure 3) if $(i) d=0,(i i)$ for each $i \in N$, $\operatorname{asp}_{i}(S, d)=e(i)$, and $(i i i) A(S, d)=A^{*}$. Let $\mathcal{B}_{\text {ord }}$ denote the set of all such problems in $\mathcal{B}$. 


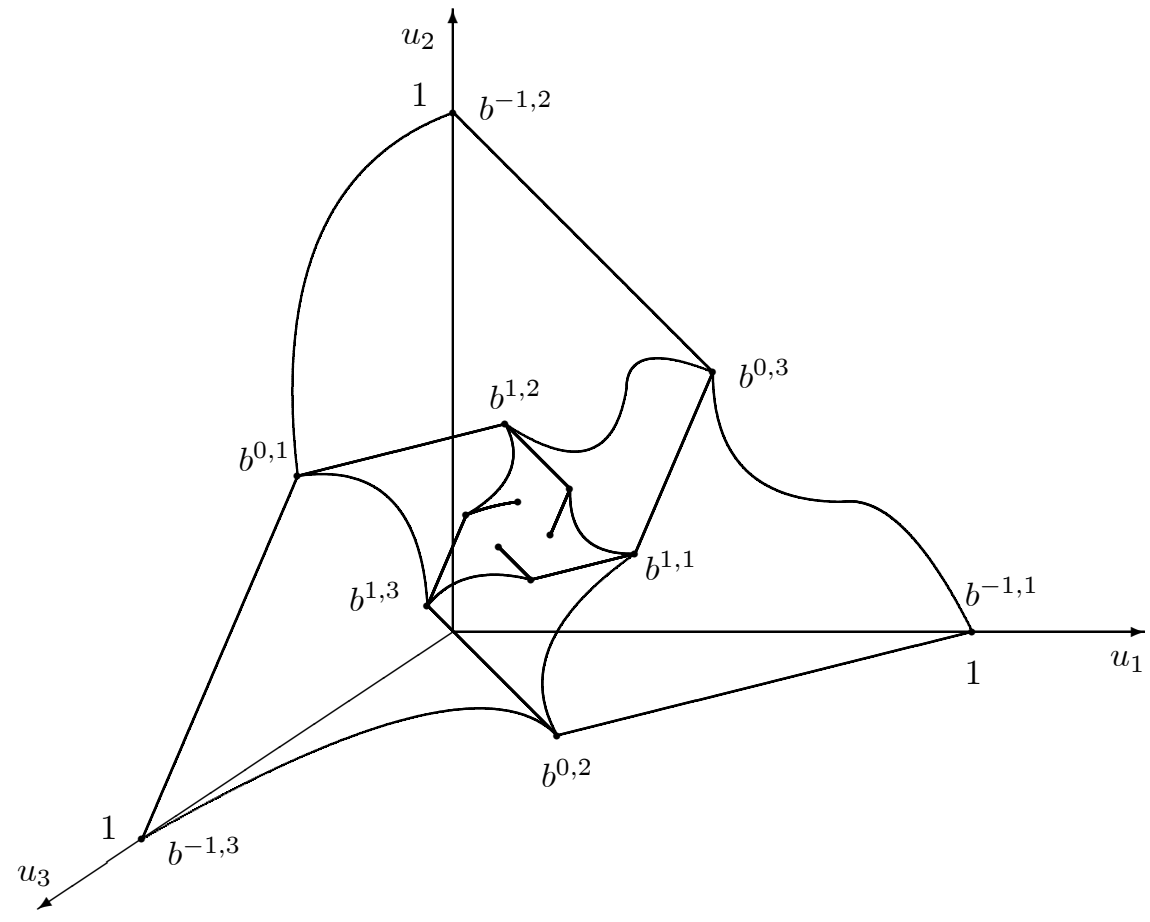

Figure 3: A typical ordinally normalized problem.

The following result states that for each $(S, d) \in \mathcal{B}$ there are order-preserving transformations of the agents utilities $\left(f_{i}\right)_{i \in N}=f \in \mathcal{F}$ such that the transformed problem $(f(S), f(d))$ is in $\mathcal{B}_{\text {ord }}$. Furthermore, the transformation is unique on the individually rational part $I(S, d)$ of $(S, d)$.

Proposition. (Kıbrıs, 2002) The subclass $B_{\text {ord }}$ ordinally spans B. Moreover, $B_{I} \cap B_{\text {ord }}$ is an ordinal basis of $B_{I}$.

This result implies that to define an individually rational and ordinally invariant bargaining rule, it suffices to describe the rule on the class of ordinally normalized problems, $\mathcal{B}_{\text {ord }}$. The ordinal invariance axiom then determines the solution to an arbitrary problem in relation to the rule's solution to an ordinally equivalent problem in $\mathcal{B}_{\text {ord }}$.

\subsection{Bargaining rules}

A bargaining rule $F: \mathcal{B} \rightarrow \mathbb{R}_{+}^{N}$ assigns each bargaining problem $(S, d) \in \mathcal{B}$ to a feasible payoff profile $F(S, d) \in S$. The following bargaining rule plays a central role in our analysis. 
For each $(S, d) \in \mathcal{B}$, the Shapley-Shubik bargaining rule, $S h: \mathcal{B} \rightarrow \mathbb{R}^{N}$ selects the limit of the brace points (equivalently the limit of the sequence $\left\{p^{n}(S, d)\right\}_{n \in \mathbb{N}}$ ) as the solution:

$$
S h(S, d):=\lim _{n \rightarrow \infty} p^{n}(S, d)
$$

A bargaining rule $F$ is Pareto optimal if it assigns each bargaining problem $(S, d) \in \mathcal{B}$ to a Pareto optimal payoff profile, $F(S, d) \in P(S, d)$. It is symmetric if given $(S, d) \in \mathcal{B}$, for each $\pi \in \Pi, \pi(S)=S$ and $\pi(d)=d$ implies that for each $i, j \in N F_{i}(S, d)=F_{j}(S, d)$. A bargaining rule $F$ is ordinally invariant if for each $(S, d) \in \mathcal{B}$ and $f \in \mathcal{F}, F(f(S), f(d))=$ $f(F(S, d))$. The scale invariance axiom of Nash is a weaker version which only allows positive affine transformations.

A bargaining rule $F$ is independent of irrelevant alternatives (IIA) if for each $(S, d)$, $\left(S^{\prime}, d\right) \in \mathcal{B}, F(S, d) \in S^{\prime} \subseteq S$ implies $F(S, d)=F\left(S^{\prime}, d\right)$. It is independent of irrelevant alternatives other than the aspiration points (IIA-aspiration) if for each $(S, d)$, $\left(S^{\prime}, d\right) \in \mathcal{B}, F(S, d) \in S^{\prime} \subseteq S$ and for each $i \in N$ asp $i(S, d) \in S^{\prime}$ imply $F(S, d)=F\left(S^{\prime}, d\right)$. It is independent of irrelevant alternatives other than the brace (IIA-brace) if for each $(S, d),\left(S^{\prime}, d\right) \in \mathcal{B}, F(S, d) \in S^{\prime} \subseteq S$ and $\operatorname{br}(S, d) \subset S^{\prime} \operatorname{imply} F(S, d)=F\left(S^{\prime}, d\right)$. It is independent of irrelevant alternatives other than the extended brace (IIAextended brace) if for each $(S, d),\left(S^{\prime}, d\right) \in \mathcal{B}, F(S, d) \in S^{\prime} \subseteq S$ and $A(S, d) \subset S^{\prime}$ imply $F(S, d)=F\left(S^{\prime}, d\right)$.

\section{Results}

In the introduction, we stated that there is no ordinally invariant bargaining rule that satisfies the other three axioms of Nash (1950). Weakening the IIA axiom in this list to $I I A$ - extended brace (that is, restricting IIA to only apply to ordinally normalized problems) however, changes this result. The Shapley-Shubik rule satisfies the property. Moreover, there are many ordinally invariant, Pareto optimal, and symmetric bargaining rules that satisfy IIA-extended brace. The following is the example of such a rule.

Example 1 Let $G^{1}=\left[(0,0,0),\left(\frac{1}{3}, \frac{1}{3}, \frac{1}{3}\right)\right]$ and $G^{2}=\left(\left(\frac{3}{4}, \frac{1}{4}, 0\right),\left(1, \frac{1}{2}, 0\right)\right)$ be two monotone 


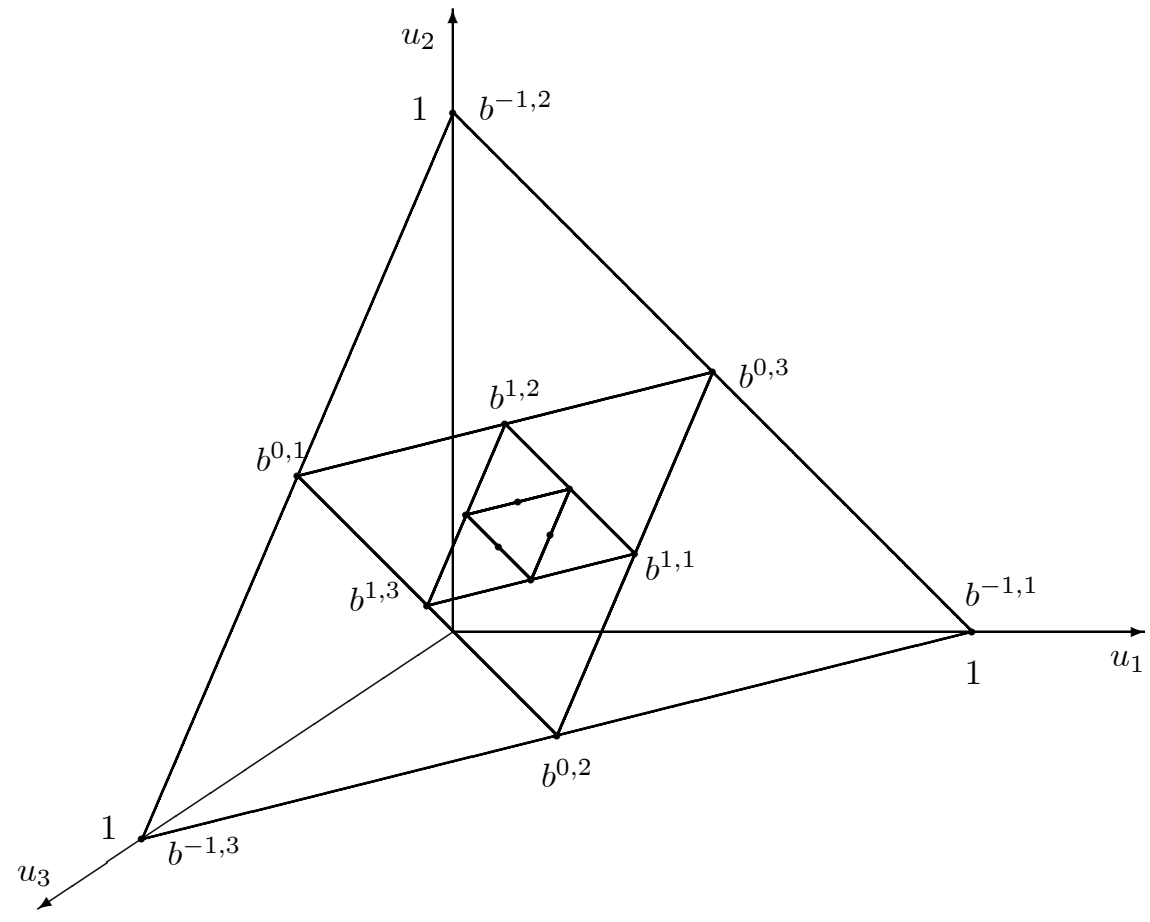

Figure 4: Constructing $C^{*}$.

paths. Let $F^{*}$ be a bargaining rule which, for each $(S, d) \in \mathcal{B}_{\text {ord }}$, is defined as:

$$
F^{*}(S, d)=\left\{\begin{array}{cc}
P(S, d) \cap G^{2} & \text { if } P(S, d) \cap G^{2} \neq \emptyset, \\
P(S, d) \cap G^{1} & \text { otherwise. }
\end{array}\right.
$$

To find the solution of $F^{*}$ to each $(S, d) \in \mathcal{B}$, (i) normalize $(S, d)$ to an $\left(S^{\prime}, d^{\prime}\right) \in \mathcal{B}_{\text {ord }}$, (ii) find $F^{*}\left(S^{\prime}, d^{\prime}\right)$ by the above method, and (iii) transform $F^{*}\left(S^{\prime}, d^{\prime}\right)$ back to $(S, d)$ by using the inverse of the normalization functions.

By definition $F^{*}$ is ordinally invariant, Pareto optimal, and satisfies IIA-extended brace. We will next prove that this rule is indeed symmetric. First note that if $(S, d) \in \mathcal{B}_{\text {ord }}$ is a symmetric problem, $P(S, d) \cap G^{2}=\emptyset$. Next, let $(S, d) \in \mathcal{B} \backslash \mathcal{B}_{\text {ord }}$ be a symmetric problem. Let $f \in \mathcal{F}$ be such that $(f(S), f(d)) \in \mathcal{B}_{\text {ord }}$. Note that $f$ is uniquely defined on $I(S, d)$ and by symmetry of $(S, d)$, satisfies $f_{1}=f_{2}=f_{3}$. Therefore, $I(f(S), f(d))$ is symmetric. This implies that $P(f(S), f(d)) \cap G^{2}=\emptyset$. So in both cases, the solution is determined via $G^{1}$. Then, since $G^{1}$ is a symmetric path, $F^{*}$ satisfies symmetry for any $(S, d) \in \mathcal{B}$.

However, the rule $F^{*}$ as well as any rule that satisfies the above properties must coincide with the Shapley-Shubik rule on a large subclass of $\mathcal{B}_{\text {ord }}$ which can be defined as follows: Let 
$d^{*}=0$ and $S^{*}=\operatorname{comp}\left\{\operatorname{conv}\{e(1), e(2), e(3)\} \mid d^{*}\right\}$. Let the construct $C^{*}$ be defined as

$$
C^{*}=\bigcup_{n=-1}^{\infty} \bigcup_{i=1}^{3}\left[b^{n, i}\left(S^{*}, d^{*}\right), b^{n, i+1}\left(S^{*}, d^{*}\right)\right]
$$

with the convention that for $i=3, i+1=1$ (See Figure 5). Note that the construct $C^{*}$ is the minimal symmetric extension of the normalized extended brace $A^{*}$. A bargaining problem $(S, d) \in \mathcal{B}$ is restricted and ordinally normalized if

1. $(S, d) \in \mathcal{B}_{\text {ord }}$

(i.e. $(S, d)$ is ordinally normalized) and

2. for each $x \in C^{*}$ and $y>x, y \notin S$

(i.e. $S$ does not contain profiles that Pareto dominate $C^{*}$ ).

Let $\mathcal{B}_{\text {rest-ord }}$ denote the class of all restricted and ordinally normalized problems. Finally, a bargaining problem $(S, d) \in \mathcal{B}$ is restricted if it is ordinally equivalent to a restricted and ordinally normalized problem $\left(S^{\prime}, d^{\prime}\right) \in \mathcal{B}_{\text {rest-ord. }}$ Let $\mathcal{B}_{\text {rest }}$ denote the class of all restricted problems.

Proposition 2 Let $F$ be a bargaining rule that satisfies ordinal invariance, Pareto optimality, symmetry, and IIA-extended brace. Then F coincides with the Shapley-Shubik rule on the class of restricted bargaining problems $\mathcal{B}_{\text {rest }}$.

Proof. Let $F$ be a bargaining rule satisfying these four axioms. Let $(S, d) \in \mathcal{B}_{\text {rest }}$. We will show that $F(S, d)=S h(S, d)$. By ordinal invariance of the two rules, we can assume without loss of generality that $(S, d) \in \mathcal{B}_{\text {rest-ord }}$. Let $x^{*}=\left(\frac{1}{3}, \frac{1}{3}, \frac{1}{3}\right)$. Since $\mathcal{B}_{\text {rest-ord }} \subset \mathcal{B}_{\text {ord }}$, $A(S, d)=A^{*}$ and $S h(S, d)=x^{*}$.

Let $T=\cup_{\pi \in \Pi} \pi(S)$. Note that $T$ is a symmetric bargaining problem and that $x^{*} \in$ $P(T, d)$. Then by Pareto optimality and symmetry of $F, F(T, d)=x^{*}$ and therefore, $F(T, d) \in S$. Now note that $(T, d) \in \mathcal{B}_{\text {rest-ord }}$. This implies that $A(T, d)=A^{*}$ and therefore, $A(T, d) \subset S$. This observations together show that the pair $(S, d),(T, d)$ satisfy the conditions of IIA-extended brace. Then, by applying this axiom, we get $F(S, d)=F(T, d)=$ $S h(S, d)$. 
Note that the class $\mathcal{B}_{\text {rest-ord }}$ is not dense in $\mathcal{B}_{\text {ord }}$. Therefore, there are continuous bargaining rules other than the Shapley-Shubik rule that satisfy ordinal invariance, Pareto optimality, symmetry, and IIA-extended brace. We next show that if the independence requirement is strenghtened to IIA-brace, a unique rule survives.

Theorem 3 The Shapley-Shubik rule uniquely satisfies ordinal invariance, Pareto optimality, symmetry, and independence of irrelevant alternatives other than the brace.

Proof. It is straightforward to show that $S h$ satisfies these four axioms. Let $F$ be a bargaining rule that also satisfies them. Let $(S, d) \in \mathcal{B}$. We will show that $F(S, d)=$ $S h(S, d)$. By ordinal invariance of the two rules, we can assume without loss of generality that $(S, d) \in \mathcal{B}_{\text {ord }}$. Let $x^{*}=\left(\frac{1}{3}, \frac{1}{3}, \frac{1}{3}\right)$ and note that $S h(S, d)=x^{*}$.

Let $T=\cup_{\pi \in \Pi} \pi(S)$. Note that $T$ is a symmetric bargaining problem and that $x^{*} \in$ $P(T, d)$. Then by Pareto optimality and symmetry of $F, F(T, d)=x^{*}$ and therefore, $F(T, d) \in S$. Also note that for each $\pi \in \Pi \pi(d)=d$ and for each $n \in \mathbb{N}$ and $i \in N$, $\pi\left(b^{n, i}(S, d)\right)=b^{n, \pi(i)}(\pi(S), \pi(d))$. Thus for each $\pi \in \Pi, \operatorname{br}(S, d)=\operatorname{br}(\pi(S), d)$. This implies that $\operatorname{br}(T, d)=\operatorname{br}(S, d) \subset S$. These observations together show that the pair $(S, d),(T, d)$ satisfy the conditions of IIA-brace. Then, by applying this axiom, we get $F(S, d)=F(T, d)=S h(S, d)$.

IIA-brace is the strongest axiom of its kind that ordinally invariant, Pareto optimal, and symmetric bargaining rules satisfy. To see this, we will introduce the following axiom which is slightly stronger than IIA-brace: it drops the feasibility requirement for one arbitrary point in the brace. A bargaining rule $F$ is independent of irrelevant alternatives other than brace minus 1 (IIA-brace-1) if for each $(S, d),\left(S^{\prime}, d\right) \in \mathcal{B}, F(S, d) \in S^{\prime} \subseteq S$ and $\operatorname{br}(S, d) \backslash\left\{b^{n, i}(S, d)\right\} \subset S^{\prime}$ for some $i \in N$ and $n \in \mathbb{N}$ imply $F(S, d)=F\left(S^{\prime}, d\right)$. The next example demonstrates a case in which the Shapley-Shubik rule violates this stronger axiom. ${ }^{8}$

Example 4 Let $y(i)=24 e(i)$ for each $i \in N$. Let $d=0$ and $S=\operatorname{comp}\{\operatorname{conv}\{y(1), y(2), y(3)\}$ | d\}. Note that $S h(S, d)=(8,8,8) . \quad S^{\prime}$ is constructed as demonstrated in Figure 4: let

\footnotetext{
${ }^{8}$ In this example, the feasible set contracts in a way that all left out profiles are the ones that assign Agent 1 a smaller payoff compared to the other two agents. Therefore, it is only intuitive that Agent 1 be better-off as result of such a contraction.
} 


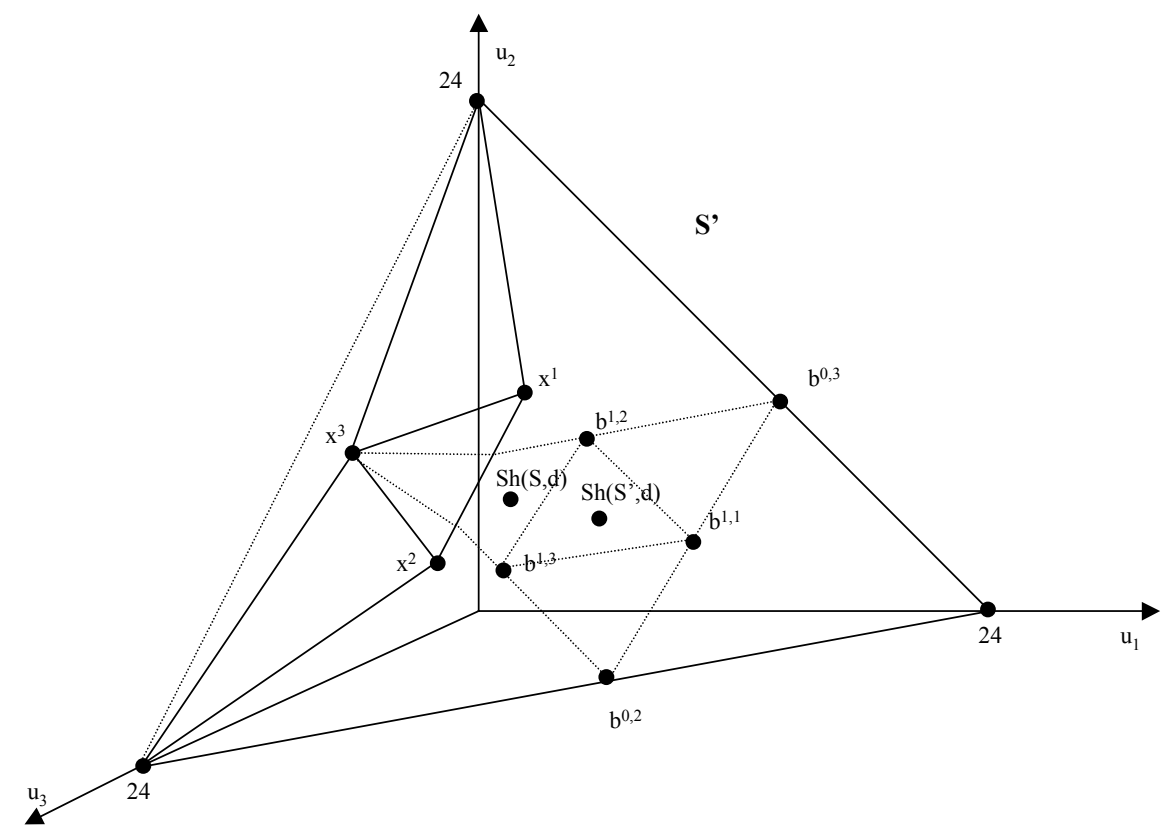

Figure 5: The Shapley-Shubik solutions to $(S, d)$ and $\left(S^{\prime}, d\right)$ are different.

$x^{1}=(6,12,6), x^{2}=(6,6,12)$, and $x^{3}=(0,10,10)$. Let $Q^{1}=\operatorname{conv}\left\{y(1), y(2), x^{1}\right\}, Q^{2}=$ $\operatorname{conv}\left\{y(1), x^{1}, x^{2}\right\}, Q^{3}=\operatorname{conv}\left\{y(1), y(3), x^{2}\right\}, Q^{4}=\operatorname{conv}\left\{y(3), x^{2}, x^{3}\right\}, Q^{5}=\operatorname{conv}\left\{x^{1}, x^{2}, x^{3}\right\}$, and $Q^{6}=\operatorname{conv}\left\{y(2), x^{1}, x^{3}\right\}$. Let $S^{\prime}=\operatorname{comp}\left\{\cup_{k=1}^{6} Q^{k} \mid d\right\}$. Note that $S^{\prime} \subset S$ and asp $\left(S^{\prime}, d\right)=$ $\operatorname{asp}(S, d)$. Note that all brace points of the original problem $(S, d)$ except $b^{0,1}(S, d)=(0,12,12)$ remain feasible in the smaller problem. Therefore, IIA-brace-1 requires that the solution to $\left(S^{\prime}, d\right)$ be $(8,8,8)$.

It is straightforward to check that the first six brace points of $\left(S^{\prime}, d\right)$ are $b^{0,1}=(0,10,10)$, $b^{0,2}=(14,0,10), b^{0,3}=(14,10,0), b^{1,1}=(14,5,5), b^{1,2}=(9,10,5)$, and $b^{1,3}=(9,5,10)$. Then, it follows from the definition of $S h\left(S^{\prime}, d\right)$ that $b_{1}^{1,2}=9<S h_{1}\left(S^{\prime}, d\right)$. Since $S h_{1}(S, d)=$ $8, \operatorname{Sh}\left(S^{\prime}, d\right) \neq S h(S, d)$.

The following proposition follows from this example and Theorem 3.

Proposition 5 No bargaining rule simultaneously satisfies ordinal invariance, Pareto optimality, symmetry, and IIA-brace-1. 
Proof. Any bargaining rule that satisfies IIA-brace-1 also satisfies IIA-brace. Therefore, by Theorem 3, the only bargaining rule that can satisfy the above list of axioms is Sh. However, by Example 1, Sh violates IIA-brace-1.

Also note that, in Example 1, the aspiration points of $(S, d)$ remain feasible in the smaller problem $\left(S^{\prime}, d\right)$. That is, the Shapley-Shubik rule violates the IIA-aspiration axiom of Roth (1977). ${ }^{9}$ The following proposition follows from this observation and Theorem 3.

Proposition 6 No bargaining rule simultaneously satisfies ordinal invariance, Pareto optimality, symmetry, and IIA-aspiration.

Proof. Define the following axiom which combines the requirements of both IIA-aspiration and IIA-brace: a bargaining rule $F$ is independent of irrelevant alternatives other than the aspiration and the brace (IIA-aspiration-brace) if for each $(S, d),\left(S^{\prime}, d\right) \in \mathcal{B}$, $F(S, d) \in S^{\prime} \subseteq S, \operatorname{br}(S, d) \subset S^{\prime}$, and for each $i \in N, \operatorname{asp}_{i}(S, d) \in S \operatorname{imply} F(S, d)=F\left(S^{\prime}, d\right)$. Note that any bargaining rule that satisfies IIA-aspiration also satisfies this axiom. Since for each $(S, d) \in \mathcal{B}_{\text {ord }}$ and $i \in N \operatorname{asp}_{i}(S, d)=e(i)$, it is straightforward to modify the proof of Theorem 3 to show that Sh uniquely satisfies ordinal invariance, Pareto optimality, symmetry, and IIA-aspiration-brace. Therefore, Sh is also the only bargaining rule that can satisfy the above list of axioms. However, by Example 1, Sh violates IIA-aspiration.

\section{References}

[1] Kalai, E. and Smorodinsky, M. (1975), "Other Solutions to Nash's Bargaining Problem", Econometrica, 43, 513-518.

[2] Kalai, E. (1977), "Proportional Solutions to Bargaining Situations: Interpersonal Utility Comparisons", Econometrica, 45, 1623-1630.

[3] Kıbrıs, Ö. (2004), "Egalitarianism in Ordinal Bargaining: the Shapley-Shubik Rule", Games and Economic Behavior, 49: 1, 157-170.

\footnotetext{
${ }^{9}$ This is not very surprising. The aspiration points (together with the disagreement point) are of significance for cardinal bargaining (since any bargaining problem can be cardinally normalized via these points). However, they do not have a similar function in ordinal bargaining.
} 
[4] Luce, R. D. and Raiffa, H. (1957), Games and Decisions, New York, John Wiley and Sons.

[5] Nash, J. F. (1950), "The Bargaining Problem", Econometrica, 18, 155-162.

[6] Roth, A. E. (1977), "Independence of Irrelevant Alternatives and Solutions to Nash's Bargaining Problem", Journal of Economic Theory, 16, 247-251.

[7] Roth, A. E. (1979), Axiomatic Models of Bargaining, Springer-Verlag.

[8] Safra, Z. and Samet, Dov (2001), "Extending Shapley's Solution to More than Three Players", working paper, at http://www.tau.ac.il/ samet/.

[9] Safra, Z. and Samet, Dov (2001), "Ordinal Solutions to Bargaining Problems", working paper, at http://www.tau.ac.il/ samet/.

[10] Shapley, L. (1969), "Utility Comparison and the Theory of Games", La Décision: Agrégation et Dynamique des Ordres de Préférence, PARIS: Editions du CNRS, 251-263.

[11] Shubik, M. (1982), Game Theory in the Social Sciences, MIT Press, Cambridge, Massachusetts, 92-98.

[12] Sprumont, Y. (2000), "A Note on Ordinally Equivalent Pareto Surfaces", Journal of Mathematical Economics, 34, 27-38.

[13] Thomson, W., Bargaining Theory: the Axiomatic Approach, book manuscript. 